\title{
Modelling and Simulation of Cratering and Ejecta Production During High Velocity Impacts
}

\author{
W. Schill ${ }^{1} \cdot$ J. V. Wasem ${ }^{2}$ (D) J. M. Owen ${ }^{2}$
}

Received: 23 September 2016 / Accepted: 18 January 2017 / Published online: 3 February 2017

(C) The authors 2017

\begin{abstract}
During an impact event from a high velocity particle (moving at speeds of around $100 \mathrm{~m} / \mathrm{s}$ or greater) a significant amount of ejecta can be formed and thrown away from the impact point at velocities on the same order as the original impactor. The amount, size distribution, and speed distribution of this ejecta vary with the impact conditions. Predicting this cratering and ejecta phenomena has applications to many areas, including solar system formation, asteroid defense considerations, and micrometeorite impacts on satellites. This paper will look at the physics and modeling of these impacts and the subsequent ejecta formation from these hypervelocity particles. Impacts are modeled using adaptive smooth particle hydrodynamics, and crater volume and ejecta characteristics are quantified and presented. Good agreement with two experimental test cases is obtained.
\end{abstract}

Keywords Hypervelocity $\cdot$ Impact $\cdot$ Cratering $\cdot$ Ejecta

J. V. Wasem

wasem2@1ln1.gov

W. Schill

wschill@ caltech.edu

J. M. Owen

owen8@1lnl.gov

1 California Institute of Technology, MC 104-44, Pasadena, CA 91125, USA

2 Lawrence Livermore National Laboratory, 7000 East Avenue, L-031, Livermore, CA 94550, USA

\section{Introduction}

Predictive science for cratering and ejecta phenomena is an integral component of multiple areas of science and engineering. In the field of solar system formation and dynamics the phenomenon has been studied extensively from a scaling-law perspective, asteroid collisions for planetary defense, and space debris and micro-meteoroid threat mitigation in the design of manned and unmanned space flight. A typical generic impact event is depicted in Fig. 1, where an impacting object of radius $a$ and density $\delta$ impacts a surface with velocity $v$. The impacted material has density $\rho$ and strength $Y$. While the actual dynamics of the impact are fairly complicated, work over many decades has shown the degree to which a range of impact phenomena can be predicted from simple combinations of these parameters (see Refs. [1-3] and references therein). Other work has concentrated on the relationship between these fundamental scaling laws and the existing experimental work (see Refs. [4-6] and references therein). However, relatively little has been published about the distribution of ejecta sizes and velocities that are created and thrown off from the direct simulation of such impacts.

We develop a computational model of crater formation using an adaptive smooth particle hydrodynamic spatial discretization scheme, implemented in the Spheral++ [7-10] computational package, to study the phenomena of cratering and ejecta formation during cratering events. We first outline the computational challenges and the approach taken to address these, along with the physical models employed in the work. We then describe the methodology by which we measure the excavated crater volume and debris field; particular interest is given to choosing the measure of ejecta fragment size. We then compute several specific examples and compare the results to experiment, 


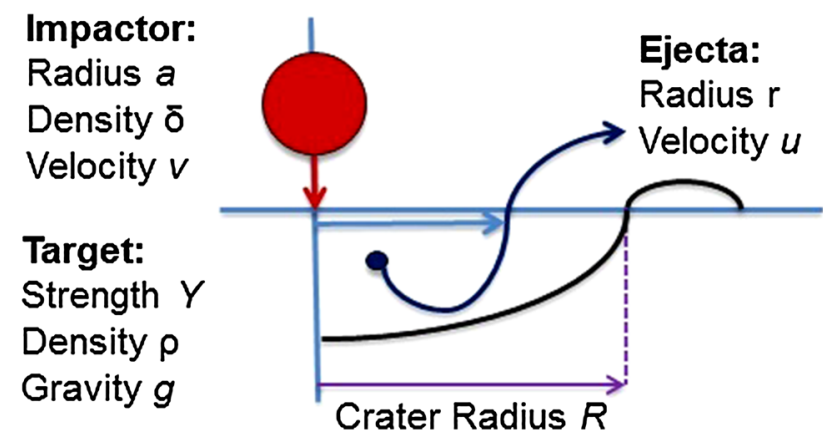

Fig. 1 A spherical impactor with radius $a$, density $\delta$, and velocity $v$ strikes a target material with strength $Y$, density $\rho$, and with gravitational acceleration $g$ and forms a crater and ejecta. The ejecta escapes the crater with a distribution of velocities $u$ and sizes $r$

where good agreement between the computational and experimental results is observed. Finally, we discuss the ejecta distributions obtained from one of the scenarios computed.

\section{Methodology}

For the modeling of the impact of high- and hyper-velocity particles the velocities and energies involved are sufficient to place the calculations in the realm of high energy density physics. Typical tools in this regime involved hydrodynamic simulations with the material discretized into a mesh. This mesh then moves in either a Lagrangian fashion (where the mesh itself moves and the material within each mesh zone remains the same) or in an Eulerian fashion (where the mesh remains constant and the material moves through the zones). One can even go further and perform re-zoning at various timepoints to smooth out mesh tangling issues in what is known as the Arbitrary Lagrangian-Eulerian (ALE) methodology. Or in the Eulerian framework one can refine the mesh dynamically to capture physically important areas, known as Eulerian Adaptive Mesh Refinement (AMR). The technology to perform these operations in a self-consistent manner has advanced by leaps and bounds over the last several decades. However, for problems concerning ejecta, meshed methods still fall short.

Standard ALE or AMR (such as CTH, used in Ref. [6]) codes typically have several problems when it comes to accurately creating and tracking ejecta. The first of these significant difficulties include capturing the physics of large deformations and fracture mechanisms that dominate crater and ejecta formation. Various models for zone damage and crushing have been utilized, with varying degrees of success, but these still tend to give incorrect answers for ejecta production, even if they correctly capture crater formation.
Following the ejecta creation, its subsequent motion out of the impact zone and into a ballistic trajectory also presents difficulties for mesh-based hydrocodes, as the size and shape of the ejecta typically depends on interface reconstruction routines. These routines will often severely distort ejecta shapes and mis-identify neighboring pieces as one, continuous, ejecta particle.

However, mesh-free methods are naturally suited to model these ejecta phenomena. Specifically we turn to a smooth-particle hydrodynamics approach [11-14]. Here the materials in the problem are treated as a series of nodes, each of which represents a certain amount of material which interacts hydrodynamically with neighboring nodes via an interpolation kernel. Because each particle is not necessarily tied computationally to its neighbors, any material ejected from the impact point can free-stream away without resorting interface reconstruction or ALE techniques. Furthermore, as each node is guaranteed to remain strictly Lagrangian, sub-scale damage models can be robustly employed which accurately represent material damage throughout the material at both macro- and microscales. To this end, we employ an Adaptive Smooth Particle Hydrodynamics (ASPH) formalism following [7, 10, $14,15]$. ASPH updates the equations of conservation of mass and momentum according to

$\rho_{i}=\sum_{j} m_{j} W_{i j}$

$\frac{D v_{i}^{\alpha}}{d t}=\sum_{j} m_{j}\left[\left(\frac{\sigma_{i}^{\alpha \beta}}{\rho_{i}^{2}}+\frac{\sigma_{j}^{\alpha \beta}}{\rho_{j}^{2}}\right) \partial_{\alpha} W_{i j}+\Pi_{i j}^{\beta \alpha} \partial_{\beta} W_{i j}\right]+g^{\alpha}$

Mass and mass density are denoted by $m_{i}$ and $\rho_{i}$ respectively. The velocity is given by $v_{i}^{\alpha}$, and the stress by $\sigma_{i}^{\alpha \beta}$. $W_{j}=W\left(r, h_{j}\right)$ is the interpolation kernel, in our case chosen to be a cubic B-spline as in $[11,13] . \Pi_{i j}^{\alpha \beta}$ is the artificial viscosity tensor as in [8] with the smoothing radius $h_{j}$ being specific to each node. We remark that the Roman indices $(i, j)$ denote nodes and the Greek indices $(\alpha, \beta)$ denote spatial dimension with Einstein's summation convention in effect. The energy is updated using the compatibly differenced methodology of [10], which explicitly enforces total energy conservation to roundoff while maintaining good adiabatic evolution properties. A full description of this algorithm is beyond the scope of this paper-we refer the interested reader to [10] for a more thorough discussion.

For the equation of state, we employ the Livermore equation of state for both granite and copper in the numerical simulations considered. We use Steinberg-Guinan[16] strength model to capture plastic deformations. Steinberg-Guinan specifies rate dependency at high strain rates for elastic perfectly plastic materials. It specifies the yield strength, $Y$, and 
the shear modulus, $G$, as a function of pressure, $P$, temperature, $T$, and compression, $\eta$. Writing these out we have

$G=G_{0}\left[1+\left(\frac{G_{P}^{\prime}}{G_{0}}\right) \frac{P}{\eta^{1 / 3}}+\left(\frac{G_{T}^{\prime}}{G_{0}}(T-300 K)\right)\right]$

and

$$
\begin{aligned}
Y= & {\left[1+\beta\left(\epsilon+\epsilon_{i}\right)\right]^{n} } \\
\times & {\left[1+\left(\frac{Y_{p}^{\prime}}{Y_{0}}\right) \frac{P}{\eta^{1 / 3}}+\left(\frac{G_{T}^{\prime}}{G_{0}}\right)(T-300 K)\right] . }
\end{aligned}
$$

Any initial plastic strain is given as, $\epsilon_{i}$ and $\nu, \beta$, and $n$ are work hardening parameters. $G_{0}$ and $Y_{0}$ are the shear modulus and yield strength at the reference state. The notation, $G_{P}^{\prime}$ specifies a derivative of $G$ with respect to $P$. Furthermore, we may require the constraint, $\left[1+\beta\left(\epsilon+\epsilon_{i}\right)\right]^{n} \leq Y_{\max }$ where $Y_{\max }$ specifies the maximum yield strength of a particular material. It is worth noting at this point that a strength model is in fact very important even in a hypervelocity impact regime. Indeed, carrying out the same calculations as we will shortly describe yields dramatically different (and wrong) answers without a correct strength model.

To model damage and resulting fragmentation, we utilize an extension of the damage model due to Ref. [17], which is in turn based on the theory presented in Ref. [18]. To briefly summarize, we define a tensor damage variable, $D_{i}^{\alpha \beta}$, which in three dimensions is a $3 \times 3$ symmetric matrix with eigenvalues bounded in the range $[0,1]$. Each eigenvalue represents the degree of damage in the direction of the associated eigenvector: a value of 0 corresponds to undamaged material, while an eigenvalue of 1 indicates that ASPH point is fully damaged in that direction. This damage is then used to directionally reduce the material strength properties such as yield, deviatoric stress, and any negative pressure.

The damage tensor $D_{i}^{\alpha \beta}$ is evolved by assigning a strainbased probability of failure to each point according to a Weibull distribution

$n(\epsilon)=k \epsilon^{m}$

describing number of flaws per volume below a strain activation threshold, $\epsilon$, with material parameters $m$ and $k$. Practically, this is implemented by considering a number of flaws, $N_{f}$, which is much greater than the total number of particles. These are randomly assigned to the particles as flaw activation strains,

$\epsilon_{i, j}^{\mathrm{act}}=\left[\frac{j}{k V}\right]^{1 / m}$

calculated by sampling the Weibull distribution in Eq. (5). Here, $i$ indexes the particle and $j$ indexes the flaw, and $V$ the volume of the material being modeled. Following the procedure outlined in [17] the flaws are seeded sequentially according to (6) as follows. Starting with $j=1$, we randomly select a Spheral particle $i$ and give it an activation flaw via (6). We then increment $j$ and randomly select a Spheral particle again (which could be the same point), give it the next flaw in the sequence, and so forth. This procedure is repeated until each point has at least one flaw, resulting in a statistical distribution of flaws following (5). If the total number of Spheral particles in the material is $N$, statistically the total number of flaws seeded will be $N_{f} \approx N \ln (N)$, and the average number of flaws per point will be $\approx \ln (N)$.

In order to evolve the damage variable we monitor the local tensor strain defined as

$\epsilon_{i}^{\alpha \beta}=\frac{\sigma_{i}^{\alpha \beta}}{E_{i}}$

where $E_{i}$ is the Young's modulus, and when any eigenvalue of this strain exceeds a flaw activation strain on the point $i$, that point begins to accumulate damage along the direction of the associated eigenvector of $\epsilon_{i}^{\alpha \beta}$. The damage is then applied to the pair-wise Spheral particle interactions such that strength interactions due to the deviatoric stress interaction, any negative pressure (i.e., tension), and so on are scaled by $1-D_{i}^{\max }$, where $D_{i}^{\max } \in[0,1]$ is the maximum eigen-value of the damage tensor $D_{i}^{\alpha \beta}$ of point $i$. The values for granite and copper as used in this paper are given in Table 1.

Note in this simple model the entirety of the physics of damage and failure for a given material are represented by the statistical probablity of failure, approximated in this approach as obeying a Weibull distribution in (5). This probability of failure is captured by two parameters in this relation: the coefficient $k$ and power-law slope $m$. These parameters can be fit from fracture experiments as described in [18], and therefore the applicability of the resulting coefficients is dependent on the range of failure types (brittle vs. ductile, high or low strain-rate, etc.) captured by those experiments. The numerical approximations we are using here roll all of those physical processes into a simple statistical probablity of failure based on strain. More sophisticated treatments are of course

Table 1 The strength and fracture material parameters

\begin{tabular}{llll}
\hline & Granite & Copper & Units \\
\hline$k$ & $1.00 \mathrm{e} 27$ & $1.00 \mathrm{e} 5$ & $\mathrm{~cm}^{-3}$ \\
$m$ & 6.2 & 2.6 & - \\
$G_{0}$ & $2.5 \mathrm{e} 11$ & $4.77 \mathrm{e} 11$ & $\mathrm{dyn} / \mathrm{cm}^{2}$ \\
$Y_{0}$ & $3.50 \mathrm{e} 10$ & $1.2 \mathrm{e} 9$ & $\mathrm{dyn} / \mathrm{cm}^{2}$ \\
\hline
\end{tabular}


possible: one could imagine trying to separate out components of the strain for different failure regimes and measuring different probabilities of failures appropriate for those regimes in experiments, resulting in multiple Weibull (or other) fits and thereby a multi-component damage model following each of those failure paths. The approach we take in this work however assumes all the possible damage mechanmisms are rolled into a single probability of failure as a function of the total strain tensor $\epsilon^{\alpha \beta}$.

We model a generic scenario of a spherical impactor of radius $a$, striking a large block (cube of size length $l$ ) target. We do not include the effects of gas or other material near the point of impact. The boundary conditions of the block are free on the surface of impact and fixed otherwise. The size of the block is carefully chosen such that it is significantly larger than the eventual crater size, and is in fact large enough that the shock waves generated by the impact and reflecting from the fixed boundaries do not affect the crater or ejecta formation. Conversely, the block size must be kept small enough to minimize computational run time at the necessary spatial resolution. We measure the crater size by diameter, measured at the original surface, the depth, measured from the original surface to the lowest point of the crater, and the volume $V$, measured by tracking the Spheral particles which have left or will leave the crater eventually based on current speed and fragmentation (described below).

A friends of friends algorithm is used along with the damage criteria above to identify the ejecta fragments. Any fully damaged material, $D=1$, loses the ability to support a tensile load. In order to automatically identify fragments we iterate over the Spheral nodes with $D<1$ and identify the neighbors of a given node that also have $D<1$. Any neighbors of those surrounding nodes are then checked to see if they also have $D<1$, and so on until all the points in a given fragment that are undamaged have been identified. This procedure results in the so-called "friends of friends" algorithm, whereby distinct fragments can be selected from a calculation automatically. If the impacted body is fractured or cracked, this procedure results in the identification of collections of nodes that are not yet fully damaged and are separated by regions that are fully damaged. We identify these as fragments and determine their velocity as an average of the Spheral node velocities, $v_{i}^{\alpha}$, of member nodes. It is worth noting that there is a significant number of nodes that are "rubblized" (i.e. completely damaged and on their own) that are representative of all of the mass that is below the scale of resolution of simulation.

This process is illustrated diagrammatically in Fig. 2. The different colors in the final picture show the largest of the fragmented pieces in a particular impact scenario. There are many choices that can be made to measure the

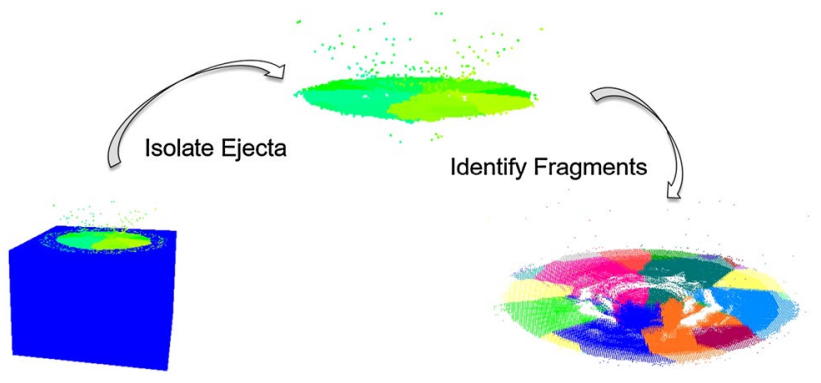

Fig. 2 In the leftmost image (at a relatively early time post-impact), we identify fragments using the damage criteria and indicate them by green color. The middle image shows the damaged material alone. We then identify ejecta fragments using the friends of friends algorithm described with different fragments denoted by different colors in the rightmost image. (Color figure online)

size of any particular ejecta fragment. A good measure for determining a meaningful sense of size for a member of a collection of particles of various shapes and sizes is that of the Sauter mean diameter [19]. The typical definition of the Sauter mean diameter proceeds as follows. Let $A_{p}$ and $V_{p}$ be the surface area and volume of the particle. Define the surface diameter

$d_{s}=\sqrt{\frac{A_{p}}{\pi}}$

and the volume diameter

$d_{v}=\left(\frac{6 V_{p}}{\pi}\right)^{1 / 3}$

Then the Sauter mean diameter is given as

$S D \equiv D[3,2] \equiv \frac{d_{v}^{3}}{d_{s}^{2}}$.

One may interpret the meaning of the Sauter mean diameter to be the diameter of a sphere that has the same volume to surface area ratio as the volume to surface ratio of the ejecta fragment. Note that for the rest of this paper any time we refer to the "ejecta size" in reference to the computational results we mean the computational determined Sauter mean diameter and not necessarily a mean particle size.

\section{Validation and Results}

Two test cases were examined to verify the effectiveness of the method and modeling paradigm. These examples were chosen to illustrate impacts in two important application materials, rock and metal. Impacts in granite are an example of impacts in rocky materials which are of 
Fig. 3 Left The crater formed during the experiment conducted by Hernandez [20]. Right The crater formed during simulation. Both images are at the same scale, and the resulting crater volumes are within $0.6 \%$

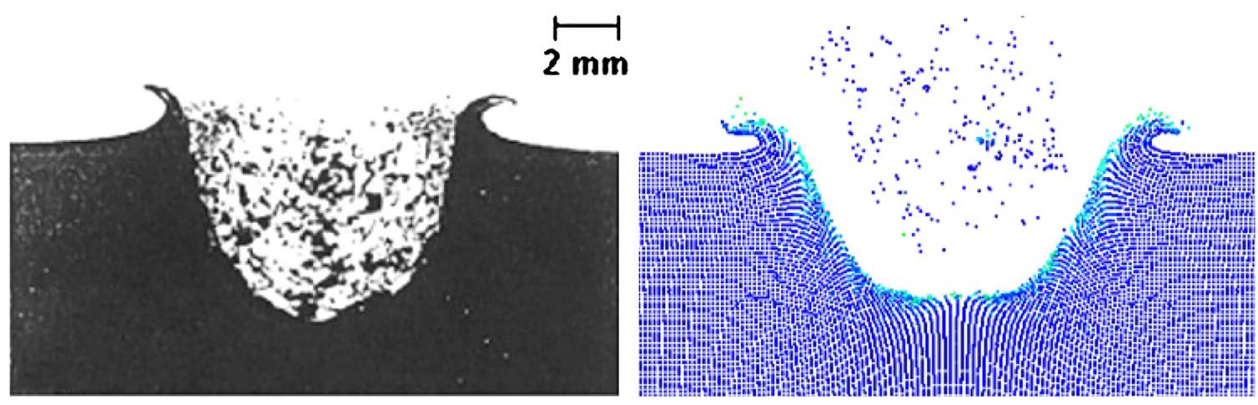

Table 2 Comparison of total crater volume between the simulation and the experiment of Ref. [20]

\begin{tabular}{ll}
\hline & Crater volume \\
\hline Experiment & $0.167 \mathrm{~cm}^{3}$ \\
Spheral calculation & $0.168 \mathrm{~cm}^{3}$ \\
$\%$ Difference & $0.6 \%$ \\
\hline
\end{tabular}

interest to the astrophysics community for solar system evolution and planetary defense. Second, an impact in copper is a representative model for the evaluation of micro-meteoroid impacts into spacecraft materials.

For impacts into metal we consider the experiment of Ref. [20]. Here a $3.18 \mathrm{~mm}$ diameter stainless steel projectile struck a copper target at $1.96 \mathrm{~km} / \mathrm{s}$. The copper target was $2.5 \mathrm{~cm}$ thick. These parameters were used in the simulation, and a visual comparison of the experimental impact crater and the simulated impact crater are shown in Fig. 3. Ejecta production in this impact regime was minimal, i.e. few significant fragments were produced, so the final crater volume is the primary metric of comparison. The volume calculated during simulation was 0.168 $\mathrm{cm}^{3}$ and the volume from the experiment was $0.167 \mathrm{~cm}^{3}$, a difference of $0.6 \%$, as tabulated in Table 2 . As one can see in in Fig. 3, both the crater diameter and depth are close to the experimental result.

Next we examine a gas gun experiment of impacts in granite, using results published in Ref. [21]. Here a $60 \mathrm{~cm}$ $\times 60 \mathrm{~cm} \times 36 \mathrm{~cm}$ block of granite was impacted by an aluminum sphere at a velocity of $7.3 \pm 0.3 \mathrm{~km} / \mathrm{s}$ with a mass and diameter of $3.029 \mathrm{~g}$ and $1.26 \mathrm{~cm}$ respectively. These parameters were replicated in the simulation. Measuring the simulated crater volume as above one obtains 780 $\mathrm{cm}^{3}$. This compares well with the crater volume described in Ref. [21], which is reported as $748 \mathrm{~cm}^{3}$ for an error of about 4 percent.

In Fig. 4 the experimental crater results are compared next to the simulation crater results (where the simulation colors indicate node damage, with red being fully damaged). One can observe similar crater sizes between the two, though the simulation crater is slightly deeper and not as wide across. The very similar excavated volume would indicate that the total energy/momentum budget of the simulation during the impact was similar to that of the experiment, but that surface irregularities in the experiment resulted in slightly different spatial distributions. Deeper into the material, where boundary effects are less prominent, one observes that similar concentric cracks in the granite have formed in both simulation and experiment. Looking at the bulk ejecta properties of this same setup,
Fig. 4 Left The crater formed during the experiment conducted by Horz [21]. Right The crater formed during simulation. Note that in both cases concentric cracks have formed at the same radius from the point of impact. Both images are at the same scale

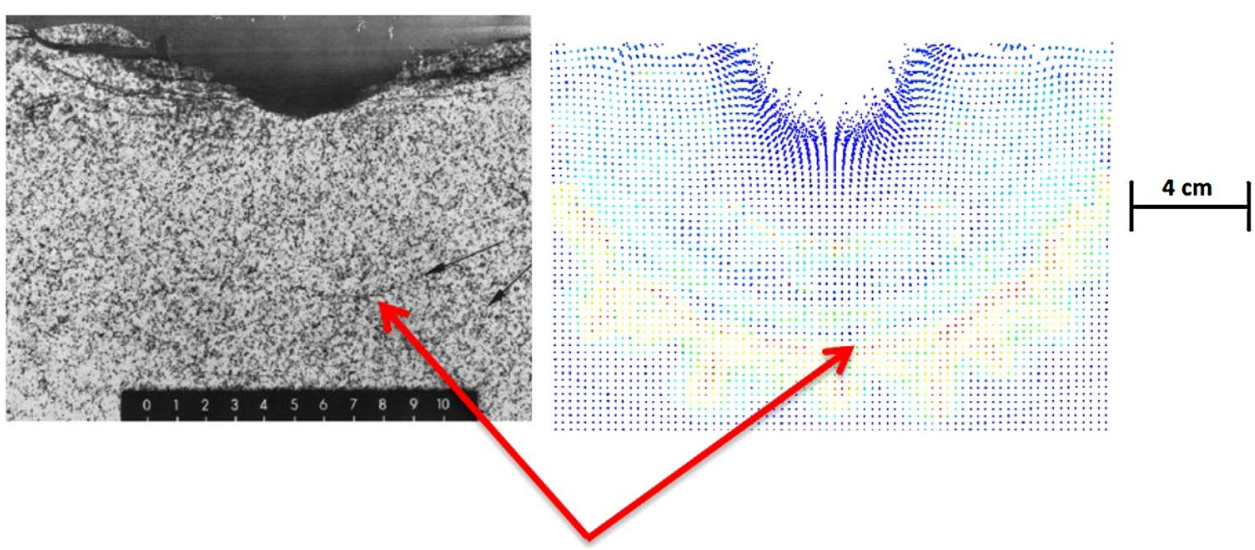

Concentric Cracks 
Table 3 Comparison of total ejecta mass and the mass of the largest fragment between the simulation and the experiment of Ref. [21]

\begin{tabular}{lll}
\hline & $\begin{array}{l}\text { Ejecta } \\
\text { Mass }\end{array}$ & $\begin{array}{l}\text { Largest } \\
\text { Fragment }\end{array}$ \\
\hline Experiment (recovered) & $1933 \mathrm{~g}$ & $124 \mathrm{~g}$ \\
Experiment (calculated) & $2024 \mathrm{~g}$ & $\mathrm{~N} / \mathrm{A}$ \\
Spheral calculation & $2007 \mathrm{~g}$ & $128 \mathrm{~g}$ \\
$\%$ Difference & $0.8 \%$ & $3.2 \%$ \\
\hline
\end{tabular}

we can compare both the total mass of the ejecta and mass of the largest fragment as described in Ref. [21]. Ref. [21] cites two ejecta total mass numbers, that recovered from the experiment and used subsequently in fragment size analysis, and that calculated from the final crater size. These numbers are compiled in Table 3. We find very good agreement with the experimental numbers, matching the largest fragment size with an error of less than $4 \%$, and matching the total ejecta production to $0.8 \%$.

Direct comparison of simulated ejecta distributions to experiment can be arduous due to the variation in initial flaw distribution that can strongly affect the outcome. Indeed, the Weibull parameters themselves along with the initial flaw distribution could be used as "knobs" to tune the answer to some extent. However, for this initial work we tuned neither the Weibull parameters nor the flaw distribution, using only the values obtained from the literature as shown in Table 1 and the initial flaw distribution as described in Sect. 2. Future work will include uncertainty quantification studies varying both aspects of the damage model.

The task of assigning a measure of size or length to ejecta fragments that are in no way uniform is challenging. To this end, we find that employing the Sauter mean diameter, as described above, does an effective job of describing the ejecta in comparison to experiment. In Fig. 5, we plot the cumulative mass distribution of both the experiment in Ref. [21] and the simulation. The line corresponding to the experiment has had all particles below our computational resolution removed to facilitate a direct comparison. We find good overall agreement, with the simulation producing relatively fewer small ejecta, and relatively more larger ejecta when compared to the experiment. It is likely that this is a simulation resolution issue, where the inherent scale provided by the smallest node in the simulation affects the final distribution. Indeed, it is worth noting here that we examined the resolution dependence of the solution by increasing the number of Spheral particles in the impacted region. Though there was no discernible effect on the crater volume or the structure of the ejecta cumulative mass distribution, the increase in resolution did add more fragments to the lower end of the mass distribution.

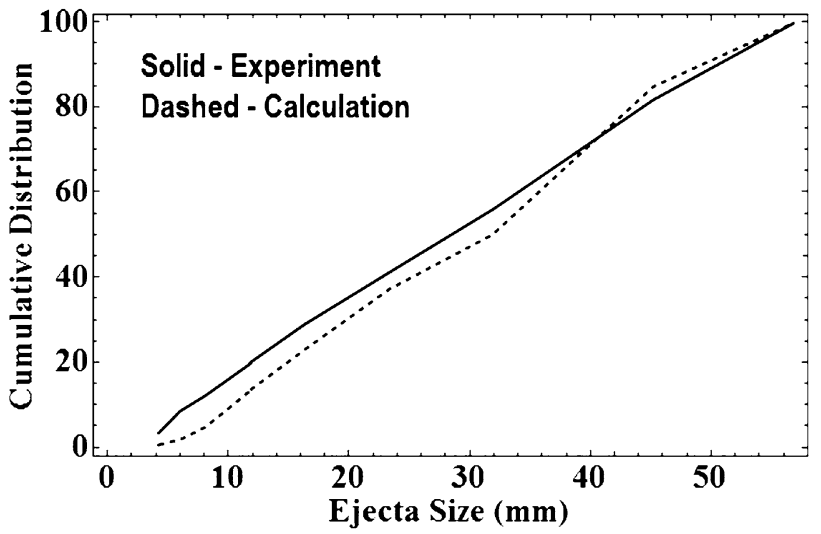

Fig. 5 The cumulative mass is the percentage of mass accounted for tracking particles with length less than or equal to a given length of ejecta fragment. The solid line is derived from Fig. 6 of Ref. [21], while the dashed line is from the Spheral calculation

Research into this effect is ongoing, but it is made difficult by the fact that the computational runtime increases as resolution to the fourth power.

A more detailed look at the number distribution of the ejecta sizes from the impact into granite is shown in Fig. 6, with the distribution PDF on top and the CDF on the bottom. Here one can see a broad distribution of particles from a few $\mathrm{mm}$ through nearly $20 \mathrm{~mm}$, with a noticeable spike in the number of particles per $\mathrm{mm}$ around $5 \mathrm{~mm}$ in size. As this size is a factor of several above our simulation resolution (meaning that each of these particles consisted of multiple nodes in the simulation) there is reason to believe this spike could be a real effect. The bin size given in the mass distribution from Ref. [21] is possibly too large to have noticed this sudden increase, and as such is not seen in the experimental data.

Broadly speaking, the clarity with which the peak at 5 $\mathrm{mm}$ occurs would indicate that there is a fundamental scale involved, either natural (based on the material properties and experimental setup) or computational (such as the resolution). As such a quick back-of-the-envelope calculation is illustrative. Using the generic impact event depicted in Fig. 1, the natural scales involved the would produce a quantity with dimensions of length would be $(\delta / m)^{-1 / 3}$ , $(\rho / m)^{-1 / 3}$, or $\left(Y / m v^{2}\right)^{-1 / 3}$ (where $m$ is the total mass of the original impactor). These scales give values of $1.04 \mathrm{~cm}$, $1.03-1.05 \mathrm{~cm}$ (depending on the granite density used), and $9.3 \mathrm{~cm}$. The last of these scales is an order of magnitude above our observed peak, indicating that it is not due to strength scaling. The scales involving density scaling are of the correct size (modulo a factor of two, which is the difference between a diameter and a radius measurement) indicating that this peak may be a simple result of the impactor mass and the target density. Further work where these 

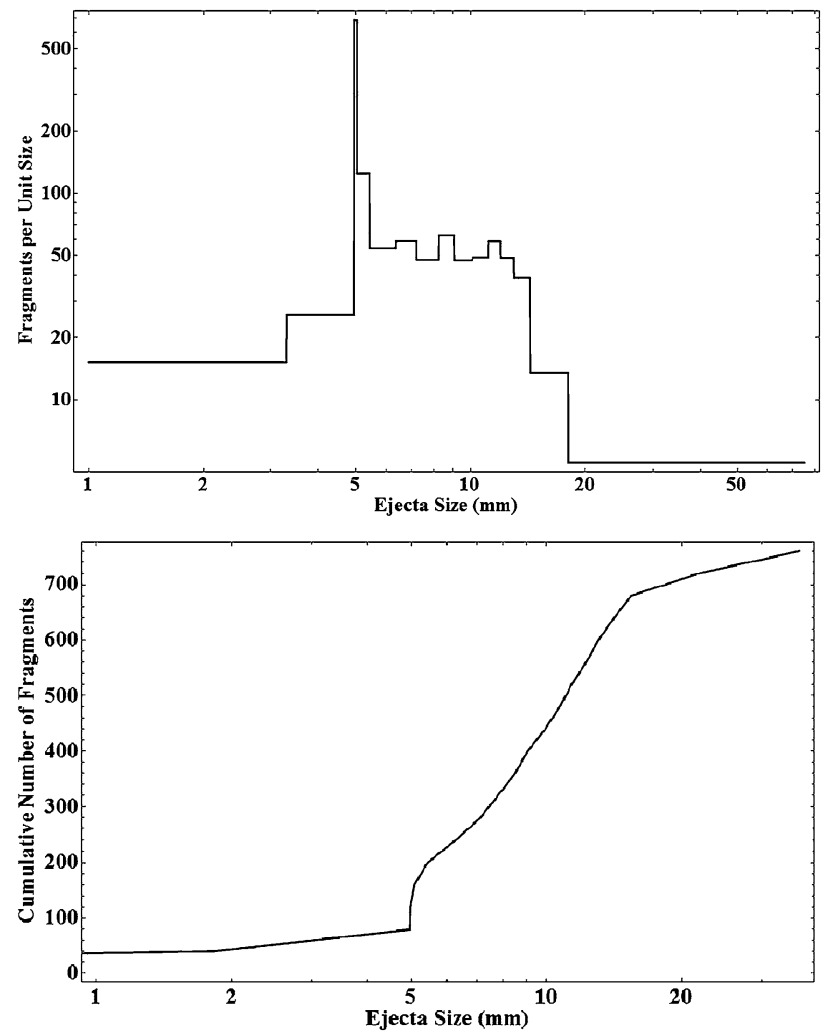

Fig. 6 Size distributions PDF (top) and CDF (bottom)

values were artificially altered could examine this hypothesis, along with variations in mesh size of the damage model flaw distribution to elucidate numerical effects.

The velocity distributions of the ejecta are shown in Fig. 7, with the distribution PDF on top and the CDF on the bottom. A large portion of the ejecta fragments are moving at less than $2.0 \mathrm{~m} / \mathrm{s}$ with a scattering of ejecta moving at higher speeds, about an order of magnitude below the impactor velocity. In fact, a significant portion of the fragments are traveling at speeds so slow that they may not exit the crater. That is, there is a significant portion of fragmented pieces that are not excavated at high speed. A smaller number of fragments leave the crater at speeds in excess of $2.0 \mathrm{~m} / \mathrm{s}$, while approximately 30 fragments exit the crater at speeds greater that $100.0 \mathrm{~m} / \mathrm{s}$. However, one can see from Fig. 8 that the high velocity particles do not constitute a significant fraction of the total ejected mass.

Examining the middle of the velocity distribution, a power law behavior is evident between 0.1 and $2.0 \mathrm{~m} / \mathrm{s}$ insofar as there is a nearly linear decrease in the log-log plot. At the low end of the distribution the power law gives way to something approaching a normal distribution with a peak at $0.045 \mathrm{~m} / \mathrm{s}$. This behavior between 0.01 and 10.0 $\mathrm{m} / \mathrm{s}$ is expected from the scaling law considerations presented in Ref. [4], where the power law occurs in a regime
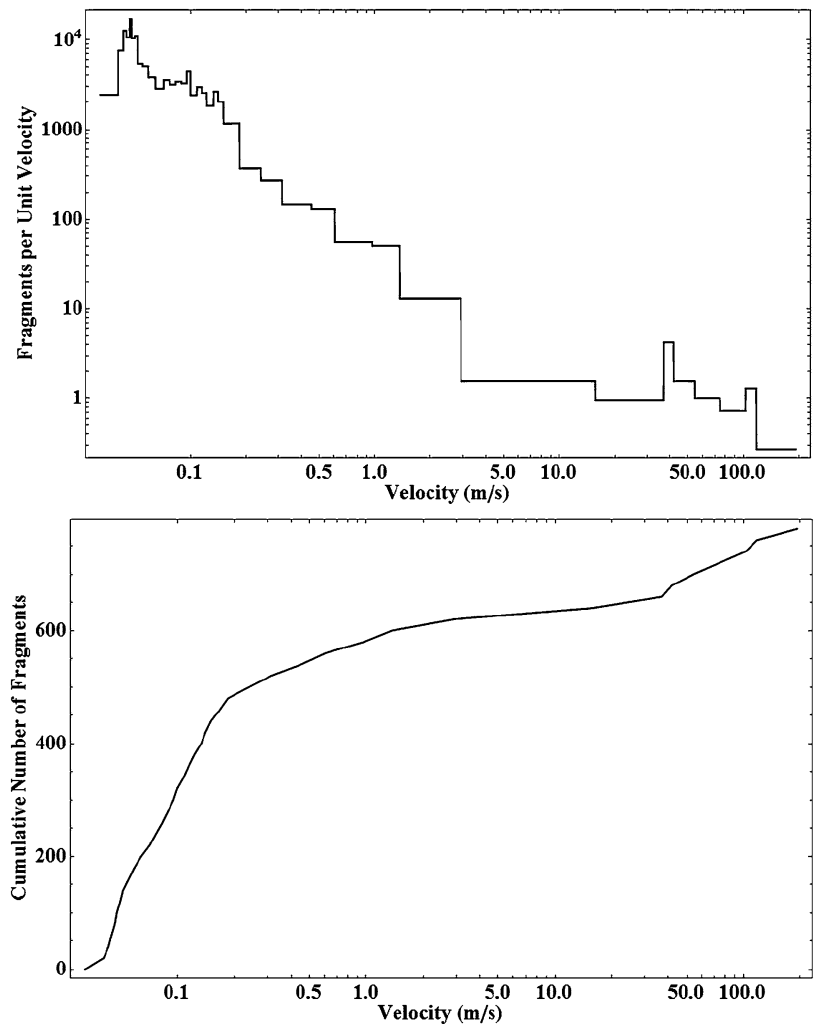

Fig. 7 Velocity distributions PDF (top) and CDF (bottom)
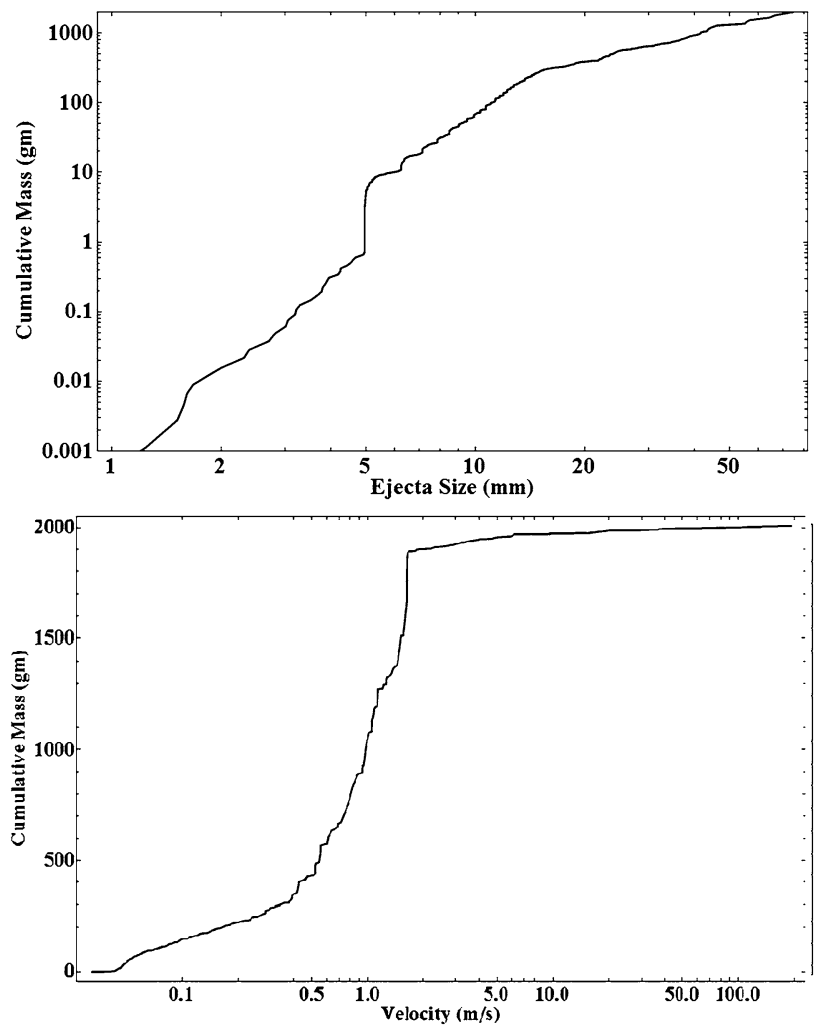

Fig. 8 Cumulative Distribution of the ejecta mass versus size (top) and velocity (bottom) 
of the problem where the impact is 'pointlike' (between 0.1 and $10.0 \mathrm{~m} / \mathrm{s}$ ), while at lower velocities the power law breaks down due to the strength effects near the edge of the crater. Taking the velocity data from between 0.1 and 100.0 $\mathrm{m} / \mathrm{s}$ for the velocity/mass distribution in Fig. 8 and fitting a power law obtains an exponent on the velocity of -1.636 . This compares very favorably with the exponent derived from Ref. [4] for competent rock of -1.65 .

Examining Fig. 8, we observe that the fastest moving ejecta constitute a very small percentage of the mass. Indeed, we note that the cumulative mass distribution nearly plateaus at around $1.0 \mathrm{~m} / \mathrm{s}$, with the fastest moving fragments (above $100.0 \mathrm{~m} / \mathrm{s}$ ) weighing much less than one gram. Looking at the results a different way, we can form the joint probability distribution for velocity and size as shown in Fig. 9. Here we can clearly see two distinct regions, one consisting of relatively small, fast-moving ejecta, and a much larger region of significantly slower but larger ejecta. The larger, slower region is what makes up the majority of the mass of the ejecta, as well as being the particles that conform most closely to the power law expected from scaling considerations for the velocity distribution. The small faster-moving particles may originate from the initial interaction region of the neck between the impactor and the slab, similar to those seen in water droplet experiments at much lower velocity [22]. Time resolved ejecta at the early times in the impact event would be needed to examine this.

It is interesting to compare the mass quantities discussed above to the mass of the impactor. The ratio of the

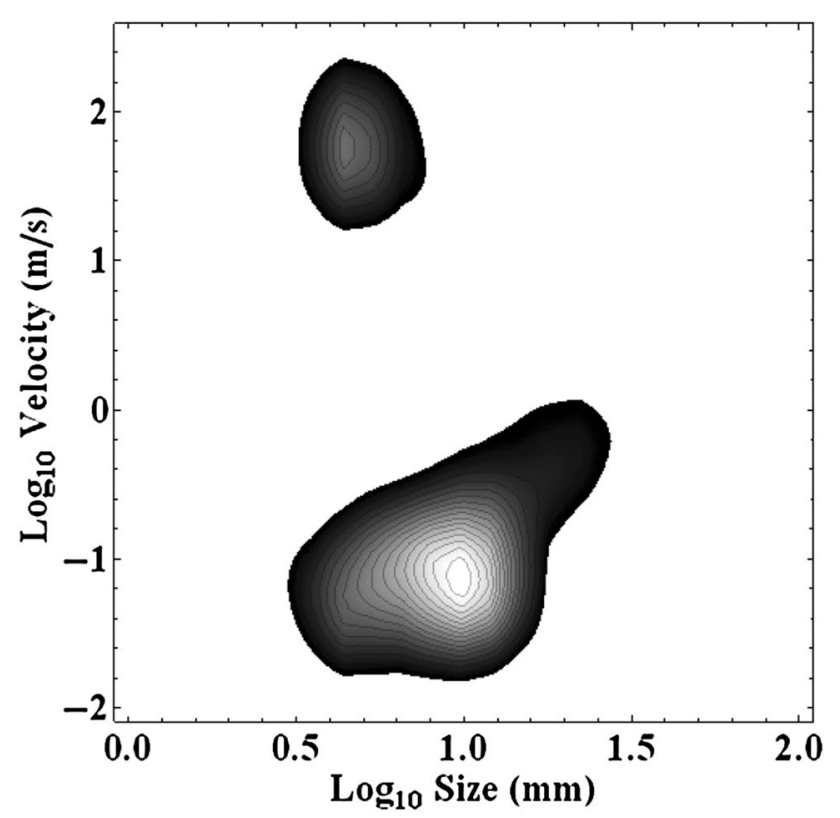

Fig. 9 Contour plot of joint distribution of velocity $(\mathrm{m} / \mathrm{s})$ and size (mm) space in log base 10 total ejecta mass to the mass of the impactor is $\approx 638$. Considering only the total mass ejected with fragments that each have less mass than the impactor, the ratio is $\approx 125$. The ratio for total mass that is moving faster than 100.0 $\mathrm{m} / \mathrm{s}$ is $\approx 0.425$. In all but the highest velocity cases the amount of material ejected is significantly more than that of the impactor. This is the origin of the $\beta$ used in planetary defense contexts. Impacts into materials less prone to fracture would likely produce less ejecta mass, but may still produce more mass than the original impactor.

\section{Conclusion}

We have modeled spherical projectiles striking several materials using adaptive smooth particle hydrodynamics with the Livermore equation of state, a Steinburg Guinan strength model, and a Grady-Kipp-Benz-Asphaug damage model. The effects of the inclusion of these various physics is very important insofar as leaving any of them out yields incorrect results as compared to experiments. It is worth noting that the use of the ASPH paradigm as compared to a more standard AMR framework gave results notably closer to the experimental values. The authors originally utilized such an AMR strategy and found it unable to effectively capture the material behavior, particularly regarding fragmentation.

We compared simulation crater volume as well as ejecta characteristics to several results from the literature and found very good agreement. We examined the velocity distributions among the ejecta resulting from the second impact. The velocity distribution varies across several orders of magnitude and the fastest moving fragments are small. The nature of the velocity distribution across several orders of magnitude in velocity conforms to what is expected from scaling law considerations presented in Ref. [4]. Additionally, the total mass of the ejecta is much larger than that of the impacting mass.

Acknowledgements We would like to thank David Dearborn, Paul Miller, Frank Graziani, Brian Pudliner, and Kirsten Howley for their valuable advice and support of this project. This work was performed under the auspices of the U.S. Department of Energy by Lawrence Livermore National Laboratory under Contract DE-AC5207NA27344, and partially funded by the Laboratory Directed Research and Development Program at LLNL under tracking code 12-ERD-005. LLNL-JRNL-703571.

\section{References}

1. Housen KR, Schmidt RM, Holsapple KA (1983) Crater ejecta scaling laws: fundamental forms based on dimensional analysis. J Geophys Res Solid Earth 88(B3):2485-2499 
2. Holsapple KA (1993) The scaling of impact processes in planetary sciences. Ann Rev Earth Planet Sci 21(1):333-373

3. Housen KR, Holsapple KA (2012) Craters without ejecta. Icarus 219(1):297-306

4. Housen KR, Holsapple KA (2011) Ejecta from impact craters. Icarus 211(1):856-875

5. Hermalyn B, Schultz PH (2010) Early-stage ejecta velocity distribution for vertical hypervelocity impacts into sand. Icarus 209:866-870

6. Hermalyn B, Schultz PH (2011) Time-resolved studies of hypervelocity vertical impacts into porous particulate targets: effects of projectile density on early-time coupling and crater growth. Icarus 216(1):269-279

7. Owen JM, Villumsen JV, Shapiro PR (1998) Adaptive smoothed particle hydrodynamics: Methodology. II. Astrophys J Suppl Ser $116(2): 155$

8. Owen JM (2004) A tensor artificial viscosity for sph. J Comput Phys. 201(2):601-629

9. Owen Jm (2009) A compatibly differenced total energy conserving form of SPH.

10. Owen JM (2014) A compatibly differenced total energy conserving form of sph. Int J Num Methods Fluids 75(11):749-774

11. Monaghan JJ (1992) Smoothed particle hydrodynamics. Araa 30:543-574

12. Libersky LD, Petschek AG, Carney TC, Hipp JR, Allahdadi FA (1993) High strain lagrangian hydrodynamics. J Comput Phys 109(1):67-75

13. Monaghan JJ (2005) Smoothed particle hydrodynamics. Rep Prog Phys 68(8): 1703
14. Gray JP, Monaghan JJ, Swift RP (2001) SPH elastic dynamics. Comput Methods Appl Mechan Eng 190(4950):6641-6662

15. Shapiro PR, Martel H, Villumsen JV, Owen JM (1996) Adaptive smoothed particle hydrodynamics with application to cosmology: methodology. Astrophys J Suppl 103:269

16. Steinberg DJ, Cochran SG, Guinan MW (1980) A constitutive model for metals applicable at high-strain rate. J Appl Phys 51(3):1498-1504

17. Brackbill JU, Eastwood JW, Benz W, Asphaug E (1995) Particle simulation methods simulations of brittle solids using smooth particle hydrodynamics. Comput Phys Commun 87(1):253-265

18. Grady DE, Kipp ME (1980) Continuum modelling of explosive fracture in oil shale. Int J Rock Mech Min Sci Geomech Abstr 17(3):147-157

19. Sauter J $(1926,1928)$ Die grössenbestimmung der in gemischnebeln von verbrennungskraftmaschinen vorhandenen brennstoffteilchen. VDI-Forschungsheft, Nr. 279 und Nr. 312, 1926,1928

20. Hernandez VS, Murr LE, Anchondo IA (2006) Experimental observations and computer simulations for metallic projectile fragmentation and impact crater development in thick metal targets. Int J Impact Eng 32(12):1981-1999

21. Hörz F (1969) Structural and mineralogical evaluation of an experimentally produced impact crater in granite. Contrib Mineral Petrol 21(4):365-377

22. Thoraval M-J, Takehara K, Etoh TG, Popinet S, Ray P, Josserand C, Zaleski S, Thoroddsen ST (2012) Von kármán vortex street within an impacting drop. Phys Rev Lett 108:264506 